\title{
Simulated Angular Response Patterns for Transhorizon Propagation'
}

\author{
J. W. Strohbehn and A. T. Waterman, Jr. \\ Contribution from the Stanford Electronics Laboratories, Stanford University, Stanford, Calif.
}

(Received August 6, 1963)

\begin{abstract}
To aid in the interpretation of rapid beam-swinging measurements as applied to tropospheric transhorizon propagation, models of the arriving wave complex have been specified, and the response of a scanning antenna array to this wave complex has been computed. Two types of models are used. One simulates a mechanism for troposcatter consisting of coherent partial reflections from layers of refractive-index discontinuity. The other simulates a mechanism of turbulent scattering. For each, the complex field at the antenna aperture is determined, for a number of specific instances, and the response of the antenna to this field is computed, in each instance, as the antenna goes through its scanning process. The results are compared with selected examples taken from transhorizon beam swinging measurements. Pertinent characteristics of each type are clearly delineated.
\end{abstract}

\section{Introduction}

In transhorizon tropospheric propagation, the wave reaching the receiving antenna, having been scattered by the atmosphere, does not necessarily arrive as a plane wave. Beam-swinging experiments [Crawford et al., 1959; Waterman, 1958; Chisholm et al., 1962] have been performed to examine the angular distribution of arriving waves. In these experiments the radio signal delivered by the receiving antenna to a receiver is measured as a function of pointing-angle of the antenna. Since antennas do not have a perfect pencil beam, the antenna response, when aimed in any one direction, is the convolution of the source distribution (of the scattered waves) with the antenna's voltage radiation pattern. In this paper, we will use the term, response pattern, to mean this convolution expressed as a function of pointing-angle of the receiving antenna. (In general, two angles, azimuth and elevation, are involved; in the numerical examples we evaluate below, only one azimuth-has been considered.)

We are concerned with multiple arriving waves and in particular wish to distinguish two cases: the response patterns for (a) the case of a small number of plane waves whose relative phases change while their individual magnitudes and directions remain substantially constant, and (b) the case of a large number of densely bunched plane waves whose relative phases change in some nonsystematic fashion. These two cases correspond to two possible models of tropospheric structure which have been invoked as possible explanations for transhorizon tropospheric scatter propagation. For simplicity, they may be

1 The research reported in this paper was made possible through the support of the U.S. Army Signal Corps under Contract DA36(039) SC-87300 of Fort Monmouth, N. J. referred to as the layer model and the turbulence model. If one assumes a model involving reflections from stratified-elevated layers [Bauer, 1956; du Castel et al., 1958], then one would expect the patterns to show a very small number of peaks - often just one - to exhibit a concentration near the great circle bearing, to have slow fading, and to change systematically in time. In contrast, if one assumes a turbulence model as proposed by Booker and Gordon, [1950], then one would expect the angular response patterns to show several peaks - occasionally quite broadened and spread over a wide angle sector - to exhibit rapid fading, and to demonstrate little continuity in time because of the large number of components in the received signal.

Stein, Johansen, and Starr [1959] have done some preliminary work in the investigation of the antenna response patterns assuming a turbulence model for the atmosphere. Their work did demonstrate that using the turbulence model one could expect single, narrow peaks, at least part of the time. However, they did not generate a group of successive antenna patterns, i.e., show the time dependence of the patterns.

The purpose of this paper is to predict theoretically the types of angular response patterns expected assuming different models for the mechanism supporting propagation as an aid in interpreting rapid beamswinging measurements. In section 2 the problem will be stated more precisely, and in sections 3 and 4 the angular response patterns will be calculated assuming a layer model and a turbulence model respectively. These two sections will show that the characteristics from the two models are extremely different, but quite similar to the characteristics intuitively expected. In section 5 there will be shown experimental examples that are extremely similar to the two types of calculated angular response patterns. The results of this work indicate 

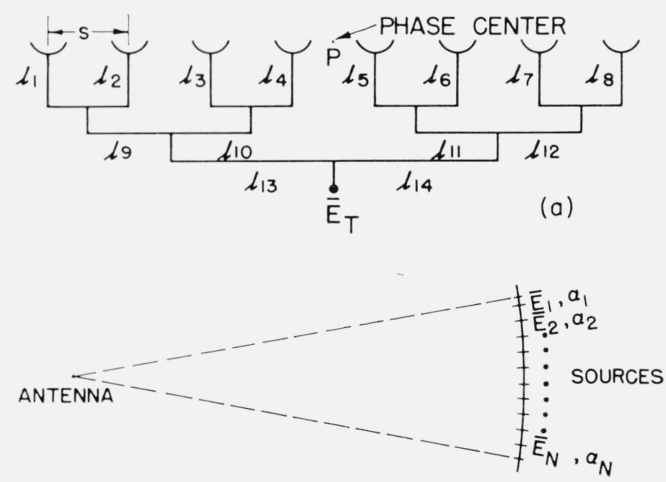

(b)

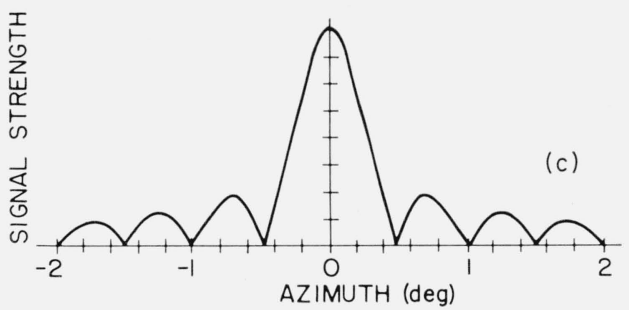

Figure 1. Antenna characteristics for theoretical model.

(a). Schematic of antenna.

(b) Schematic showing antenna and sources.

(c). Angular response of antenna to a single source on great-circle bearing.

that both mechanisms, horizontally stratified layers and turbulent eddies, are instrumental in supporting transhorizon propagation, and furthermore that the rapid beam-swinging experiment seems to be a valuable tool for differentiating between different models for the mechanism supporting propagation.

\section{Definition of Problem}

As an aid in interpreting and understanding the angular response patterns measured experimentally, a theoretical investigation of angular response patterns was performed. The antenna was patterned after that used in the Stanford experiments [Waterman, 1958; Strohbehn, 1963]. It was taken to be an eight element phased array (fig. 1a) scanned in azimuth by effectively changing the line lengths $l_{1}$ through $l_{8}$. The dimensions assumed were 1.22 $m$ spacings between adjacent elements and a wavelength of $10 \mathrm{~cm}$. Directivity of individual elements and radiative coupling between elements were neglected. The antenna was assumed to be located at the center of a circle with infinite radius on which the sources were located, so that the radiation from each source reached the antenna aperture as a plane wave. The different models were simulated by varying the sources on the circle. The antenna was scanned in the plane of figure $1 \mathrm{~b}$ over a sector including the sources.

It can be shown [Strohbehn, 1963] that the total voltage $\bar{E}_{T}$, at the antenna output is given by the following expression, if one neglects a constant phase term and considers only small angles from the broadside direction :

$$
\bar{E}_{T}(\theta, t)=2 \sum_{n=1}^{N} \bar{E}_{n}(t) \sum_{k=1}^{4} \cos \frac{2 \pi s}{\lambda}\left(k-\frac{1}{2}\right)\left(\alpha_{n}-\theta\right)
$$

where

$$
\begin{aligned}
\bar{E}_{n} & =\text { the (complex) field of the } n \text {th source. } \\
s & =\text { the spacing between antennas. } \\
\lambda & =\text { wavelength } \\
\alpha_{n}= & \text { azimuth angle of the } n \text {th source. } \\
\theta= & \text { azimuth angle of a single source for which } \\
& \text { the antenna is momentarily phased to } \\
& \text { give maximum response, i.e., the direc- } \\
\text { tion in which the antenna is aimed. } & t=\text { time. }
\end{aligned}
$$

Since we are mainly interested in the magnitude of $\bar{E}_{T}(\theta, t)$ we have:

$$
\begin{aligned}
& \left|\bar{E}_{T}(\theta, t)\right|=2\left\{\left[\sum_{n=1}^{N} E_{n}(t) \cos \phi_{n}(t) \sum_{k=1}^{4}\right.\right. \\
& \left.\cos \frac{2 \pi s}{\lambda}\left(k-\frac{1}{2}\right)\left(\alpha_{n}-\theta\right)\right]^{2}+\left[\sum_{n=1}^{N} E_{n}(t) \sin \phi_{n}(t) \sum_{k=1}^{4}\right. \\
& \left.\left.\cos \frac{2 \pi s}{\lambda}\left(k-\frac{1}{2}\right)\left(\alpha_{n}-\theta\right)\right]^{2}\right\}^{1 / 2}
\end{aligned}
$$

where

$$
\begin{aligned}
E_{n}(t) & =\text { magnitude of } \bar{E}_{n}(t) \\
\phi_{n}(t) & =\text { phase of } \bar{E}_{n}(t) .
\end{aligned}
$$

To find the angular response patterns, $\left|\bar{E}_{T}(\theta, t)\right|$, one has to calculate only $\left|\bar{E}_{T}(\theta, t)\right|$ from the source distribution $\left\{\overline{\boldsymbol{E}}_{n}(t), \alpha_{n}\right\}$, which depends on the model assumed. The angular response of the antenna to a single centrally located source is shown in figure $1 \mathrm{c}$.

\section{Layer Model}

To simulate the layer model the following assumptions were made:

1. A very small number of sources, normally less than four, are present because of the small number of layers normally present.

2. The sources remain stationary in space and constant in amplitude for the periods of time that are of interest.

3. The sources are normally quite close to the great-circle bearing as a result of the approximately horizontal structure of most layers in the atmosphere. The maximum deviation assumed is $\pm 0.5 \mathrm{deg}$.

4. The sources change phase relative to the phase center of the antenna (see fig. 1). This phase change is a result of variations in the path length between transmitter and receiver due to small changes in the reflecting point of the layer.

The angular response patterns, $\left|\bar{E}_{T}\right|$ as a function of $\theta$, for different source distributions are shown in figures 2 through 4 . The dashed lines indicate the actual positions $\left(\alpha_{n}\right)$ of the sources. In figure 2, three examples of the response to two sources with 

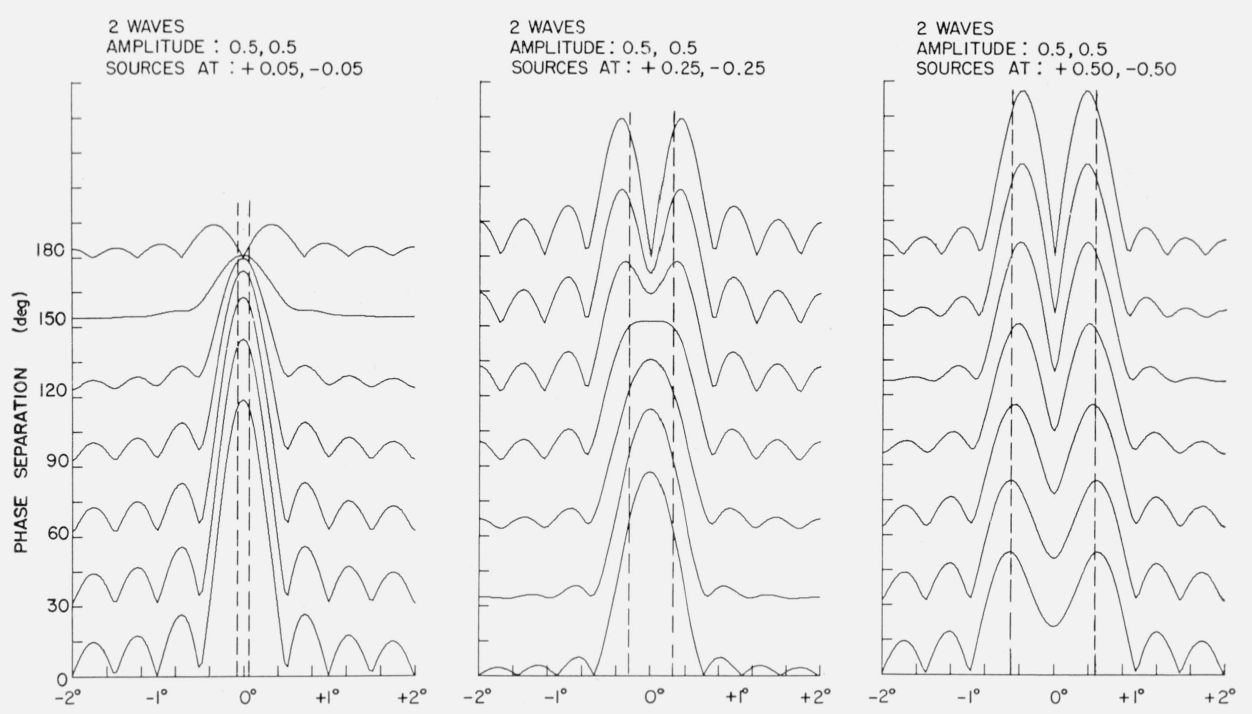

FIGURE 2. Response patierns of 8-element phased array to two coherent sources.
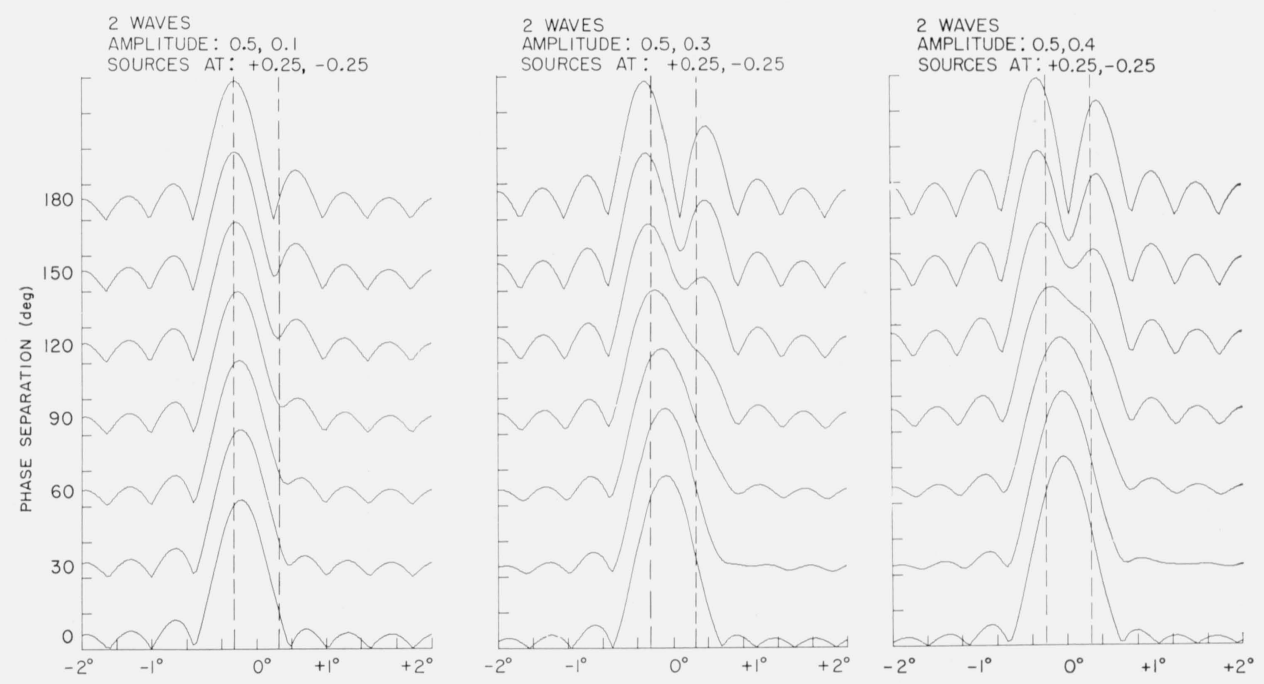

Figure 3. Response patterns of 8 -element phased array to two coherent sources with different amplitudes.
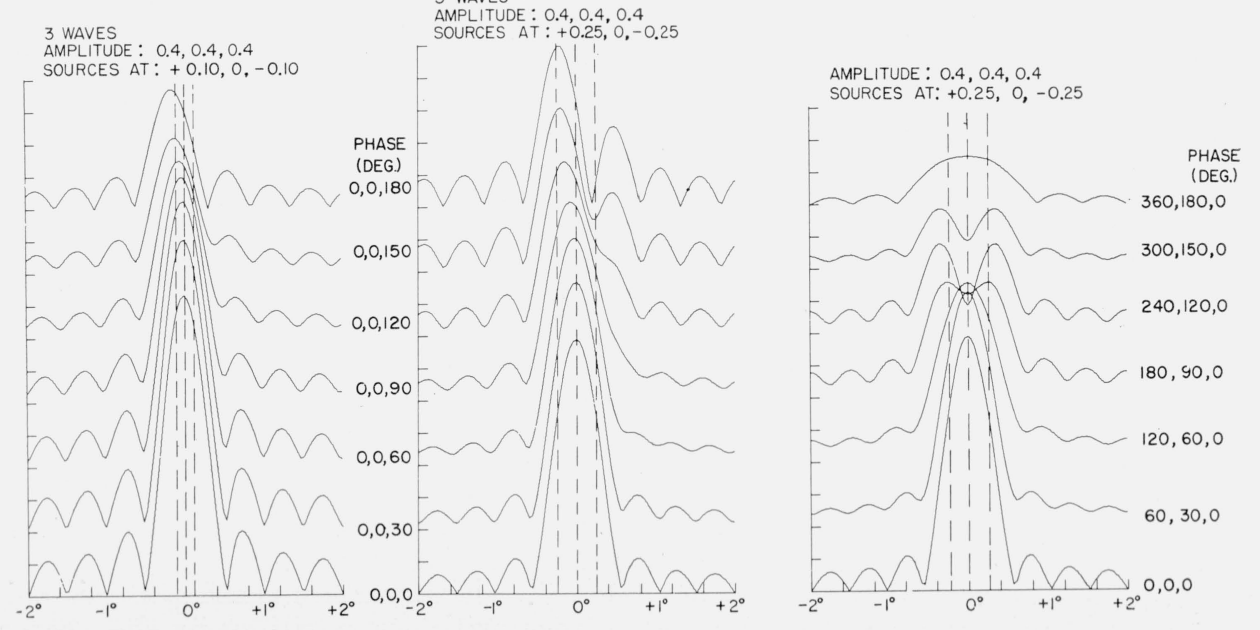

FIGURE 4. Response patterns of 8-element phased array to three coherent sources. 
equal amplitudes but with different angular separations are shown. In the lowest trace of each, the phase difference between the two sources at the phase reference of the antenna is $0 \mathrm{deg}$. In the next trace from the bottom there is a $30 \mathrm{deg}$ difference, in the third trace, $60 \mathrm{deg}$, etc., and in the last trace 180 deg. As a result, the seven consecutive traces represent the change of the angular response patterns with time, since the phase difference will normally progress with time. Only a half-cycle of sourcephase variation is shown in these figures, but if the phase difference continues to change, then an entire cycle would be seen. In figure 3, three examples of the response to two sources at a fixed angular separation, but having unequal amplitudes, are shown. The same phase progression as before is used. In figure 4 there are three examples of angular response patterns with three sources of equal amplitude, but at different angular separations, and with different phase progressions (as indicated).

There are several characteristics to notice in these patterns. Keeping the sources stationary in space and varying the phase separation between the sources can result in significant changes in the angular response patterns. In figure 2 one sees single peaks splitting into two peaks which are separated in angle by about eight times the separation between the sources. In figure 3 one sees the major peak moved a few tenths of a degree in azimuth without a major second peak appearing. Hence, one sees that phase changes between sources that are stationary in space can result in large and significant changes in the angular response patterns ${ }^{2}$ often causing the peaks to move a few tenths of a degree in azimuth in a very short time.

\section{Turbulence Model}

To approximate the turbulence model the following assumptions were made:

1. The scattered radiation reaching the receiving antenna can be considered as coming from a large number of independent sources. In our case the number chosen was 150 . (Trials with larger numbers showed 150 to be adequate for a one dimensional azimuth scan, with an 8-element array.)

2. These sources are spaced uniformly in azimuth over the sector to be covered by the antenna scan. (See fig. 1b.)

3. Each source is considered as representing a "macroscopic volume element" of turbulence scatter theory. That is, each represents the scattering from many "blobs" so that its signal has a Rayleigh amplitude distribution and a uniform phase distribution [Booker and Gordon, 1950; Staras, 1952; Villars and Weisskopf, 1954; Silverman and Balser, 1954].

4. The correlation distance of the atmospheric structure is much smaller than the macroscopic

\footnotetext{
2 The general nature of this result has long been known. Its pertinence to propagation measurements was mentioned to one of the authors by M. Jean simone. The contribution of this paper is the specific quantitative determination of the effect and its direct comparison (sec. 5) with actual measurements.
}
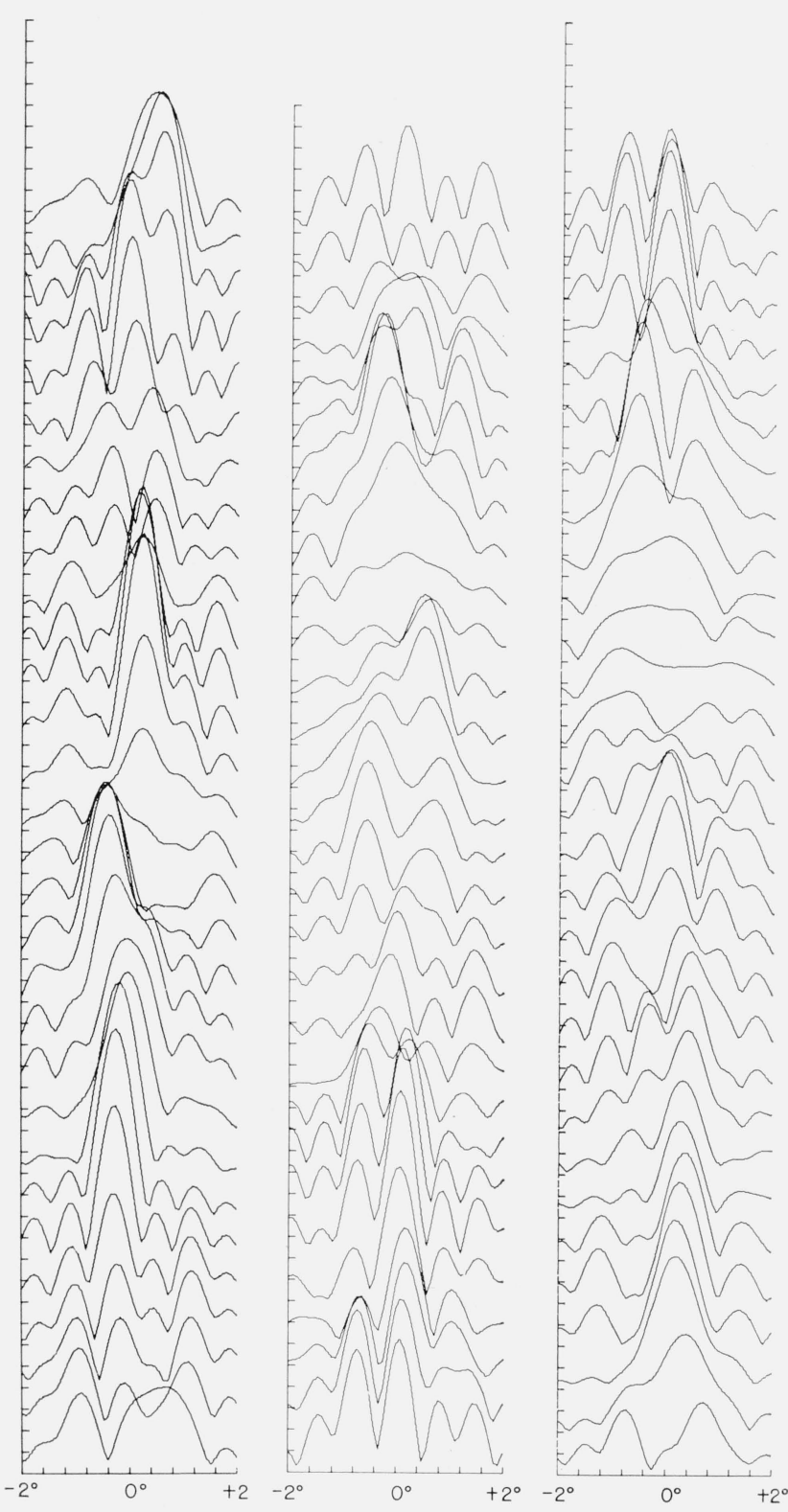

Figure 5. Response of 8-element phased array to 150 random sources.

volume element, so that each source used in the model is independent of the others.

5. Each source has a time variation corresponding to the motion of turbulent eddies and causing the resultant signal to change.

6. By having as many as 150 sources evenly spread across the sector to be scanned, there are approximately 20 sources within one beamwidth. (The 4-deg scan sector is eight times the $1 / 2$ deg beamwidth.) Thus continuity is maintained as the antenna is scanned.

7. The signal from each source is weighted in accordance with its position in the sector to take into account a scattering angle dependence (assumed to 
be inverse fourth power, but not critical) and the transmitting antenna polar diagram (sufficiently broad relative to sector size to be of minor importance).

8. Radiation from each source is held constant as the antenna scan is performed, but varied continuously from one scan to the next.

Using the assumptions listed above, angular response patterns were computed using (2). The sources were generated by using a gaussian noise generator to obtain the real and imaginary parts of each source $\bar{E}_{n}(t)$. The output from the noise generator was punched onto cards using an analogto-digital converter and IBM 1620 computer. The sampling rate could be controlled externally. The angular response patterns were then computed using an IBM 7090 computer. A sequence of 30 successive patterns in time were generated to illustrate the time dependence of the patterns. The sampling rate of the gaussian noise generator was varied until the patterns exhibited some changes over a group of 30 but still showed some continuity in time.

Three sets of angular response patterns calculated in this fashion are shown in figure 5.
There are several significant features in these patterns. First, note that the peaks are spread over a fairly wide angle sector. Second, the patterns are continually changing from single peak to double peak to broad peaks to multiple peaks. There is no one condition that seems to predominate. These features are in contrast to the ones shown in the layer model, where one expects the signal quite close to the great circle bearing, and one sees only one or two peaks which change in a systematic manner.

\section{Comparison Between Simulated and Measured Angular Response Patterns}

A beam-swinging experiment has been performed at $S$-band over a 100-mile transhorizon path [Waterman, 1958; Strohbehn, 1963]. The antenna and other parameters used in the theoretical models were taken from this experiment. Film records of the angular response patterns, spaced 0.1 sec apart in time and each covering a $4^{\circ}$ azimuthal sector, have revealed records exhibiting characteristics similar to those from both the layer model and the turbulence model. A comparison between the

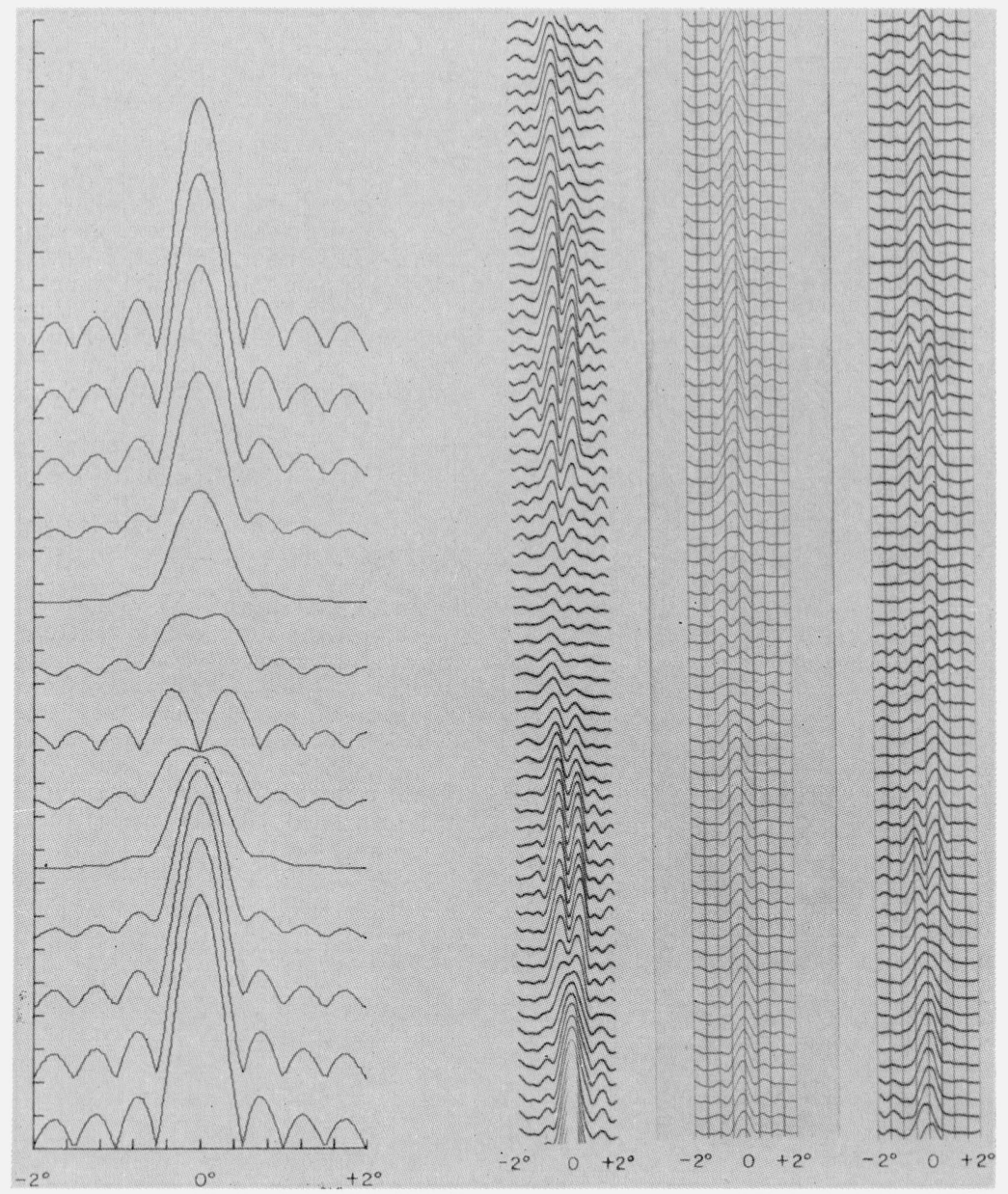

FiguRE 6. Comparison of theoretical layer response patterns with similar experimental ones. 


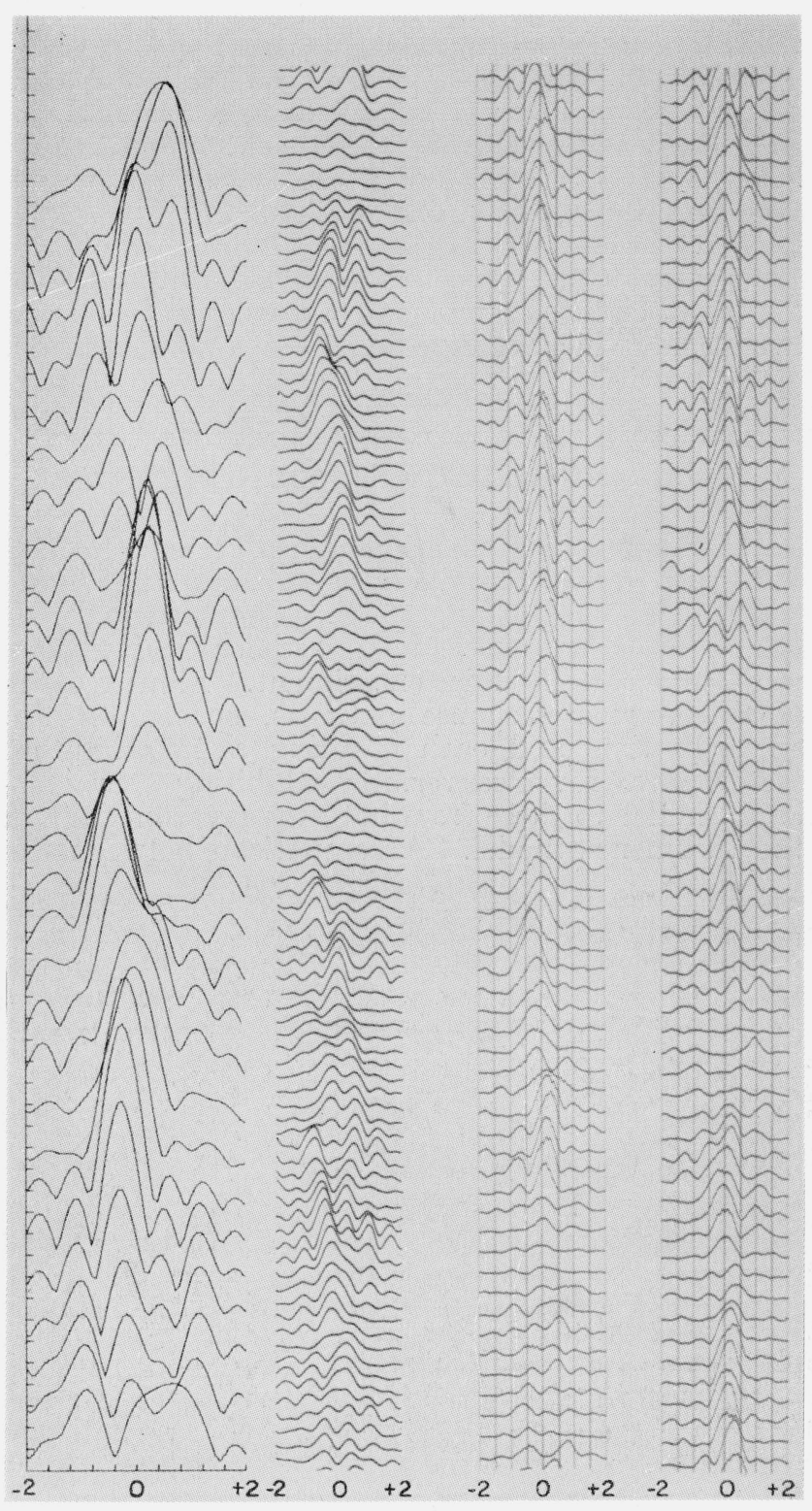

FIguRE 7. Comparison of theoretical turbulent response patterns with similar experimental ones.

simulated angular response patterns (for 2 equalamplitude sources $0.1^{\circ}$ apart) and some patterns measured on the transhorizon path is shown in figure 6 . The splitting of one peak into two, with an associated drop in signal level, and subsequent rejoining into one is clearly illustrated.

In figure 7 a similar comparison is made for the turbulence case. Here the simulated response patterns do not so literally match those measured, owing to the random nature of the variations; but the general characteristics of multiple irregular peaks, broadening, infrequent occurrence of a single unbroadened peak, and irregular motion are in evidence.

The above records are samples only, chosen to illustrate two types of situations. On the basis of similar observations scattered throughout the last few years, it appears that the two types occur about equally often on the Jackson-to-Stanford path in central California. Approximately 40 percent of all situations can be categorized as laver type and another 40 percent as turbulence type, the remaining 20 percent being transitional [Strohbehn, 1963].

\section{References}

Bauer, J. R. (24 Sept. 1956), The suggested role of stratified elevated layers in transhorizon short-wave radio propagation, TR No. 124, Lincoln Laboratory, 1-45.

Booker, H. G. and W. E. Gordon (1950), Theory of radio scattering in the troposphere, Proc. IRE 38, No. 4, 401-412.

Chisholm, J. H., W. E. Morrow, Jr., B. E. Nichols, J. F. Roche, and A. E. Teachman (1962), Properties of 400 Meps long-distance tropospheric circuits, Proc. IRE 50, No. 12, 2464-2482.

Crawford, A. B., D. C. Hogg, and W. H. Kummer (Sept. 1959), Studies in tropospheric propagation beyond the horizon, Bell System Tech. J. 38, No. 5, 1067-1178.

du Castel, F., P. Misme, A. Spizzichino, J. Voge, Réflexions Partielles Dans l'Atmosphere et Propagation à Grande Distance, Extrait des Annales des Télécommunications:

Première partie: Mesures météorologiques Tome 13, Nos. 7-8, 209-214 Juillet-Août, 1958.

Deuxième partie: Interprétation des mesures météorologiques, Tome 13, Nos. 9-11, 265-270 Septembre-Octobre, 1958 .

Troisième partie: Réflexion en milieu inhomogène, Tome 14, Nos. 1-2, 33-40 Janvier-Février, 1959.

Quatrième partie: Réflexion spéculaire et réflexion difĩuse sur des feuillets atmosphériques, Tome 15, Nos. 1-2, 38-47, Janvier-Février, 1960

Complément à la deuxième partie: Variations des dimensions des feuillets en fonction de l'altitude, Tome 15, Nos. 1-2, 48-50, Janvier-Février, 1960.

Cinquième partie: Réflexion et diffusion troposphériques des ondes radioélectriques, Tome 15, Nos. 5-6, 107-121, Mai-Juin, 1960.

Sixième partie: Les différentes composantes du champ au delà de l'horizon, Tome 15, Nos. 5-6, 122-136, Mai-Juin, 1960.

Septième partie: Note sur les phenomènes de propagation troposphérique, Tome 15, Nos. 5-6, 137-142, Mai-Juin, 1960.

Silverman, R. A. and M. Balser (1 Nov. 1954), Statistics of electromagnetic radiation scattered by a turbulent medium, Phys. Rev. 96, 560-563.

Staras, H. (1952), Scattering of electromagnetic energy in a randomly inhomogeneous atmosphere, J. of Appl. Phys. 23, No. 10, 1152-1156.

Stein, S., D. E. Johansen, and A. W. Starr (30 Sept. 1959), Final report on theory of antenna performance in scattertype reception, Hermes Electronics Co., 132-156.

Strohbehn, J. W. (Aug. 1963), Transhorizon propagation measurements and simulated angular response patterns, Stanford Electronies Labs., TR No. 2270-5, 117.

Villars, F. and V. F. Weisskopf (15 April 1954), The scattering of electromagnetic waves by turbulent atmospheric fluctuations, Phys. Rev. 94, 232-240.

Waterman, A. T., Jr., (1958), A rapid beam swinging experiment in transhorizon propagation, PGAP $\mathbf{A P}-6$, No. 4, $338-340$.

(Paper 68D2-330) 